Supporting information

\title{
Microcapsules with Distinct Dual-Layer Shells and Their Applications for the Encapsulation, Preservation and Slow Release of Hydrophilic Small Molecules
}

Yuandu Hu† and Juan Pérez-Mercader†t*

$\uparrow$ Department of Earth and Planetary Sciences, Harvard University, Cambridge, Massachusetts, United States, 02138

† Santa Fe Institute, Santa Fe, New Mexico, United States, 87501

*To whom correspondence should be addressed.

E-mail: jperezmercader@fas.harvard.edu

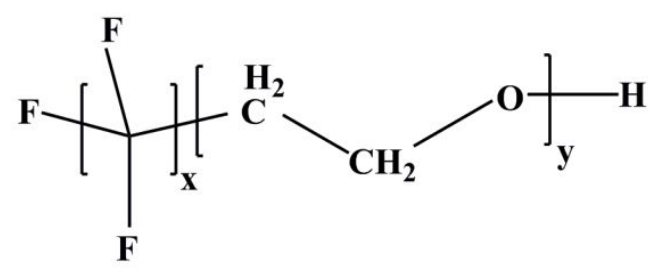

Scheme 1. Chemical structure of water soluable fluorosurfactant FS-3100.

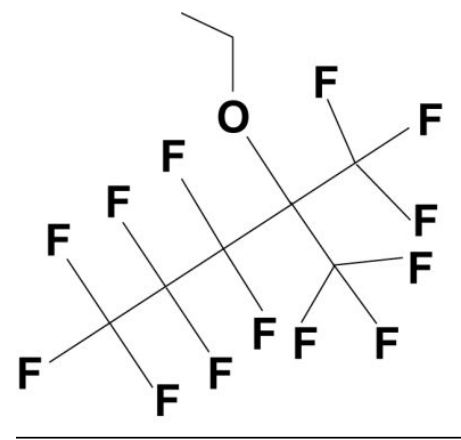

Scheme 2. Chemical structure of HFE-7500. 


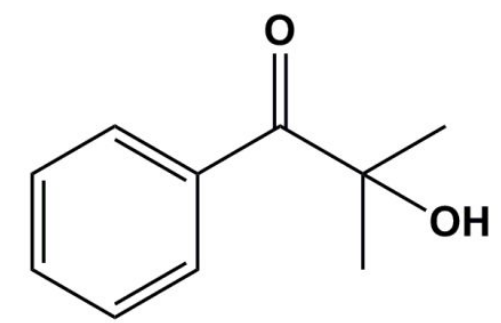

Scheme 3. Chemical structure of the photoinitiator Darocur 1173: 2-Hydroxy-2-methyl-1phenyl-propan-1-one

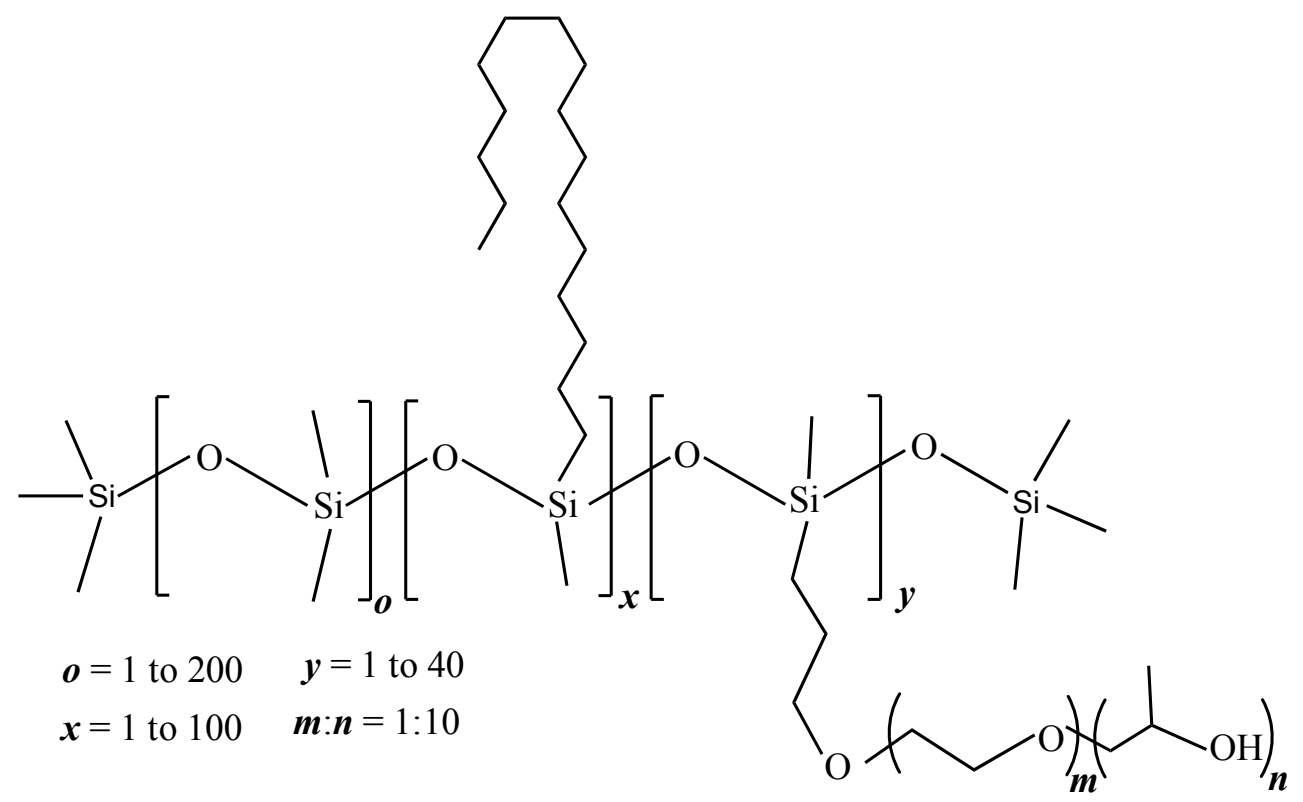

Scheme 4. Chemical formula of surfactant Abil EM90. According to previous literatures, Abil EM90 is a copolymer of Cetyl Dimethicone and an alkoxylated derivative of Dimethicone containing an average of 10 moles of ethylene oxide and 1 mole of propylene oxide. The product has an anverage molecular weight $>1000 \mathrm{Da}$; less than $5.5 \%$ of this ingredient had a molecular weight $<500 \mathrm{~g} / \mathrm{mol}$ and $9.0 \%<1000 \mathrm{~g} / \mathrm{mol}^{1}$ 

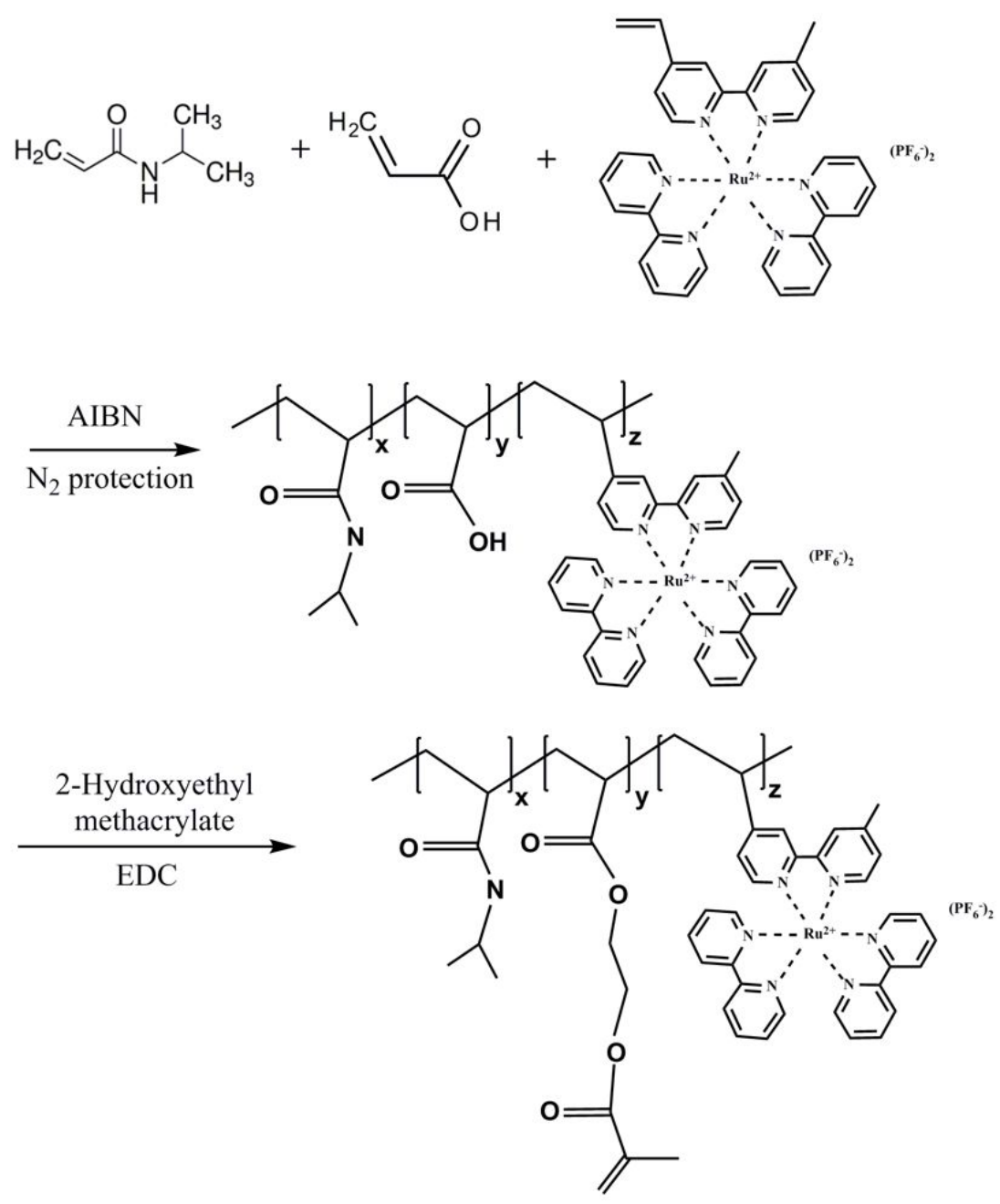

Scheme 5. Synthetic route of the ruthenium-functionalized linear polymer precursors. 

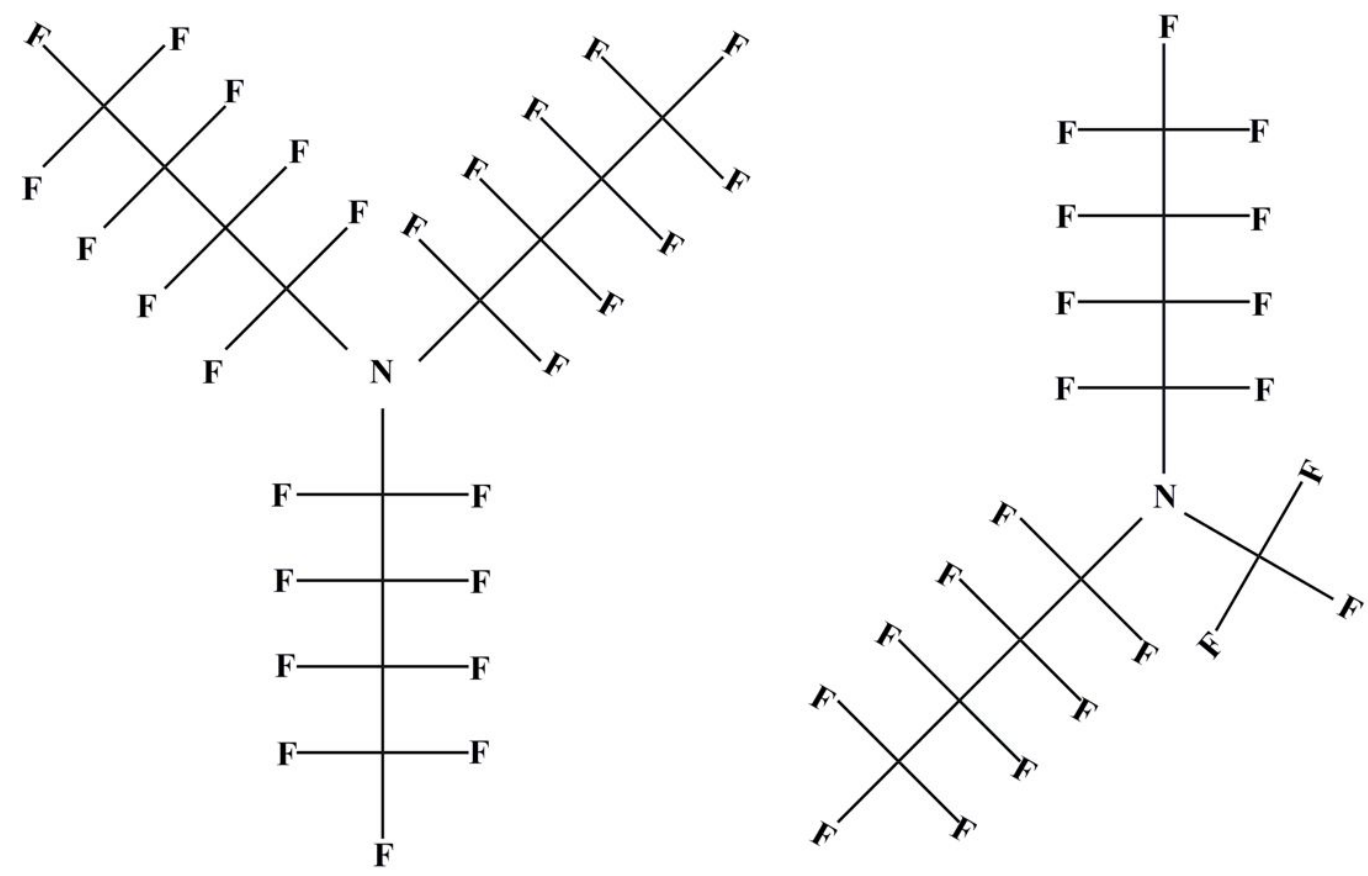

Scheme 6. Chemical structure of the Fluorinert ${ }^{\mathrm{TM}}$ FC-40 (Mixture of Perfluorotri-n-butylamine and Perfluoro-n-dibutylmethylamine, 1,1,2,2,3,3,4,4,4-Nonafluoro-N,N-bis $\quad(1,1,2,2,3,3,4,4,4$ nonafluorobutyl)butan-1-amine, 1,1,2,2,3,3,4,4,4-nonafluoro-N-(1,1,2,2,3,3,4,4,4 nonafluorobutyl) -N-(trifluoromethyl)butan-1-amine) used in the experiments. 




Figure S1 (a) - (c) Evolution of resin microcapsules with rhodamine 6G entrapped and their evolution in distilled water. (d) Illustration figure showing the structure of the resin microcapsule. 


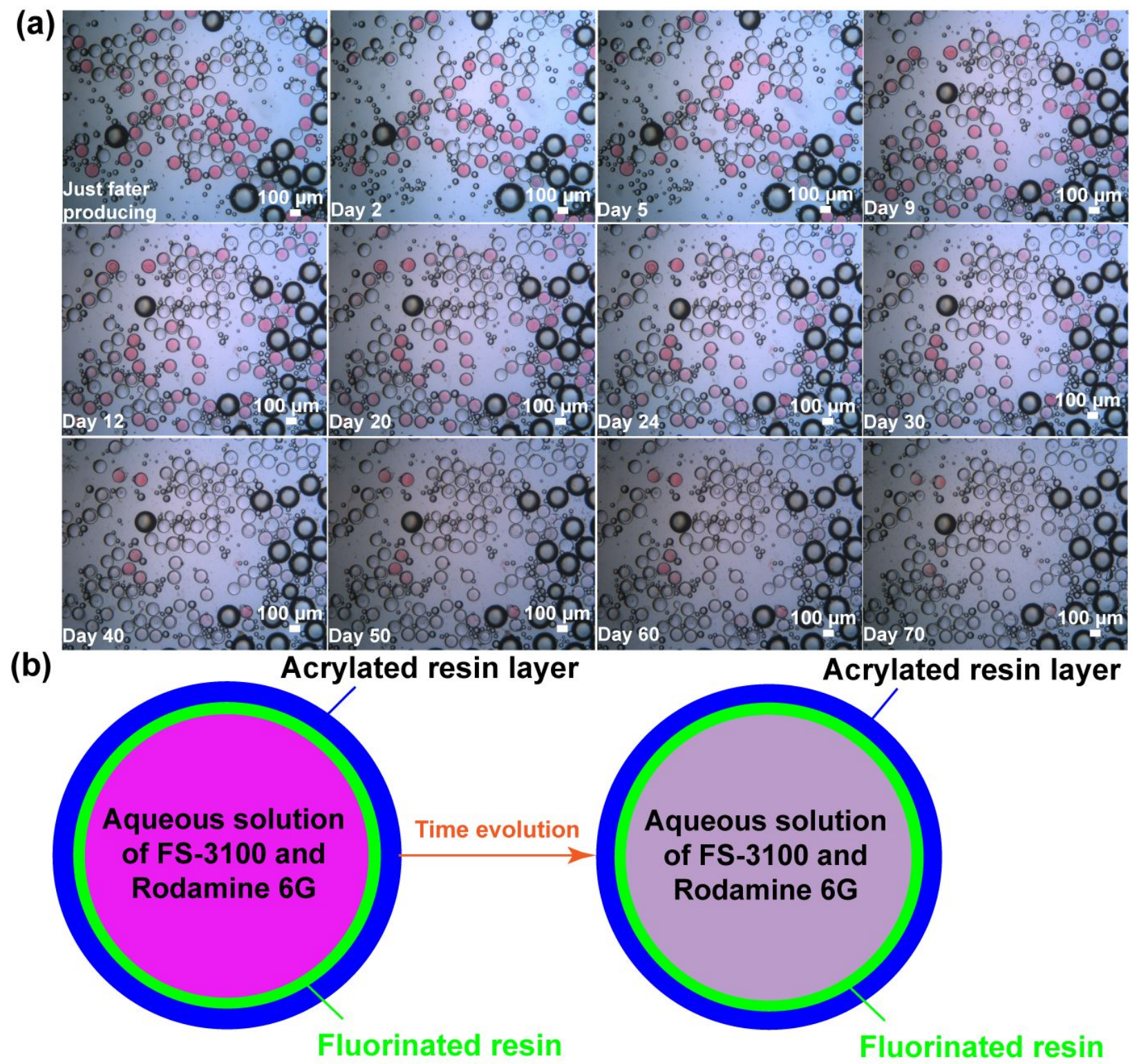

Figure S2 (a) Evolution of dual-layer shell microcapsules with photocurable resin as the external layer in $50 \mathrm{mM}$ of $\mathrm{NaCl}$ solution. (b) Illustration figure of the evolution of microcapsules in (a). 


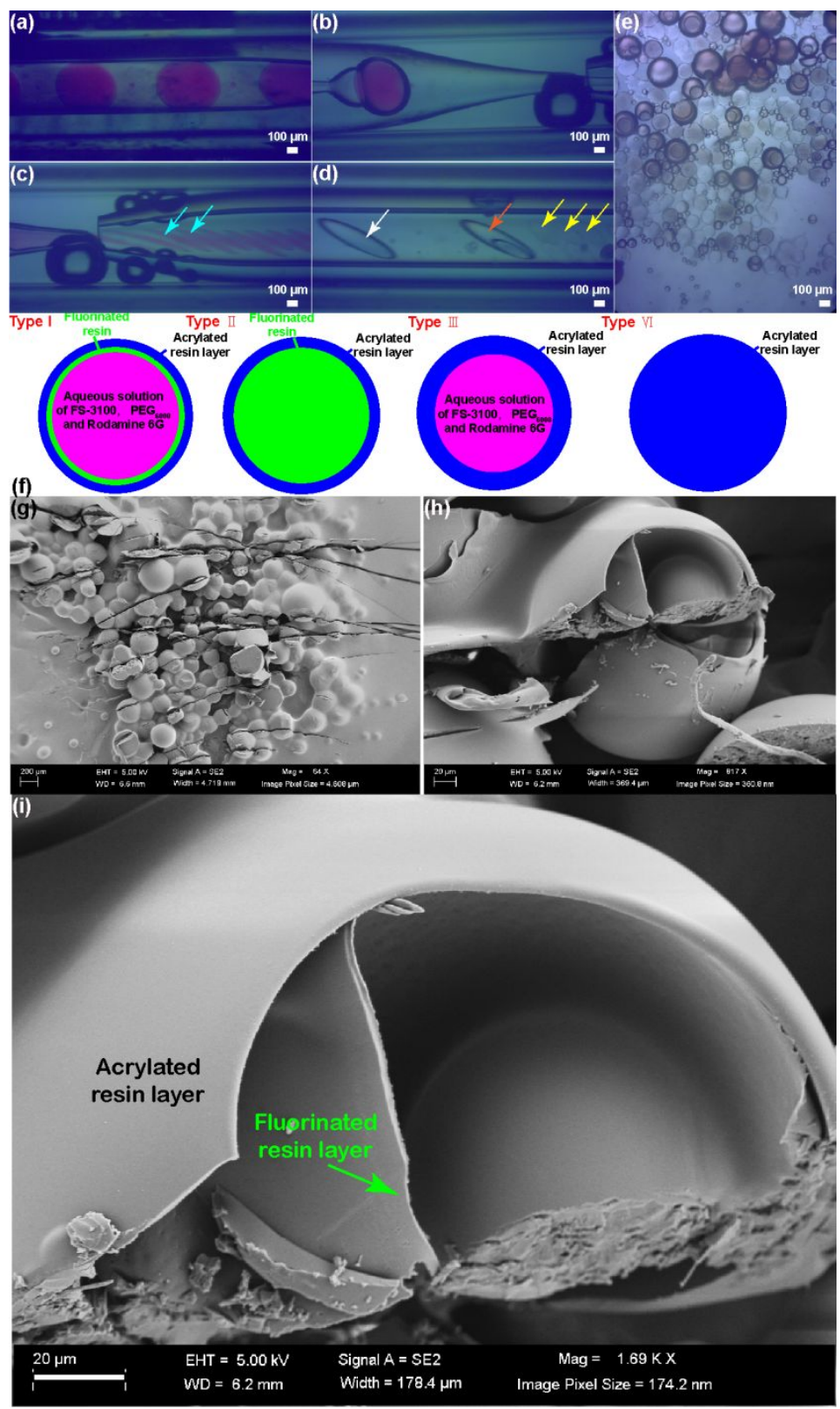

Figure S3 (a) - (c) Microfluidics processes inside microfluidic device for the generation of different emulsions: (a) Water in fluorinate oil (W/F) emulsion droplets; (b) Water in fluorinate oil in perfluorinated resin $\left(\mathrm{W} / \mathrm{F} / \mathrm{R}_{\mathrm{F}}\right)$ emulsion droplets; (c) Water in fluorinate oil in perfluorinated resin in water $\left(\mathrm{W} / \mathrm{F} / \mathrm{R}_{\mathrm{F}} / \mathrm{W}\right)$ emulsion droplets. (d) A mixture of emulsion droplets inside microfluidic device. (e) Collected mixture products. (f) Illustration figures show the different structure of the obtained products. (g) Scanning Electronic Microscopy image of the 
obtained mixture products. (h) Scanning Electronic Microscopy of a microcapsule with dual resin layers: outer acrylate resin layer and inner perfluorinated resin layer. (i) Enlarged SEM image of the resin microcapsule with dual-layer shells. The image clearly reveals that the microcapsules contains two distinct resin layers.

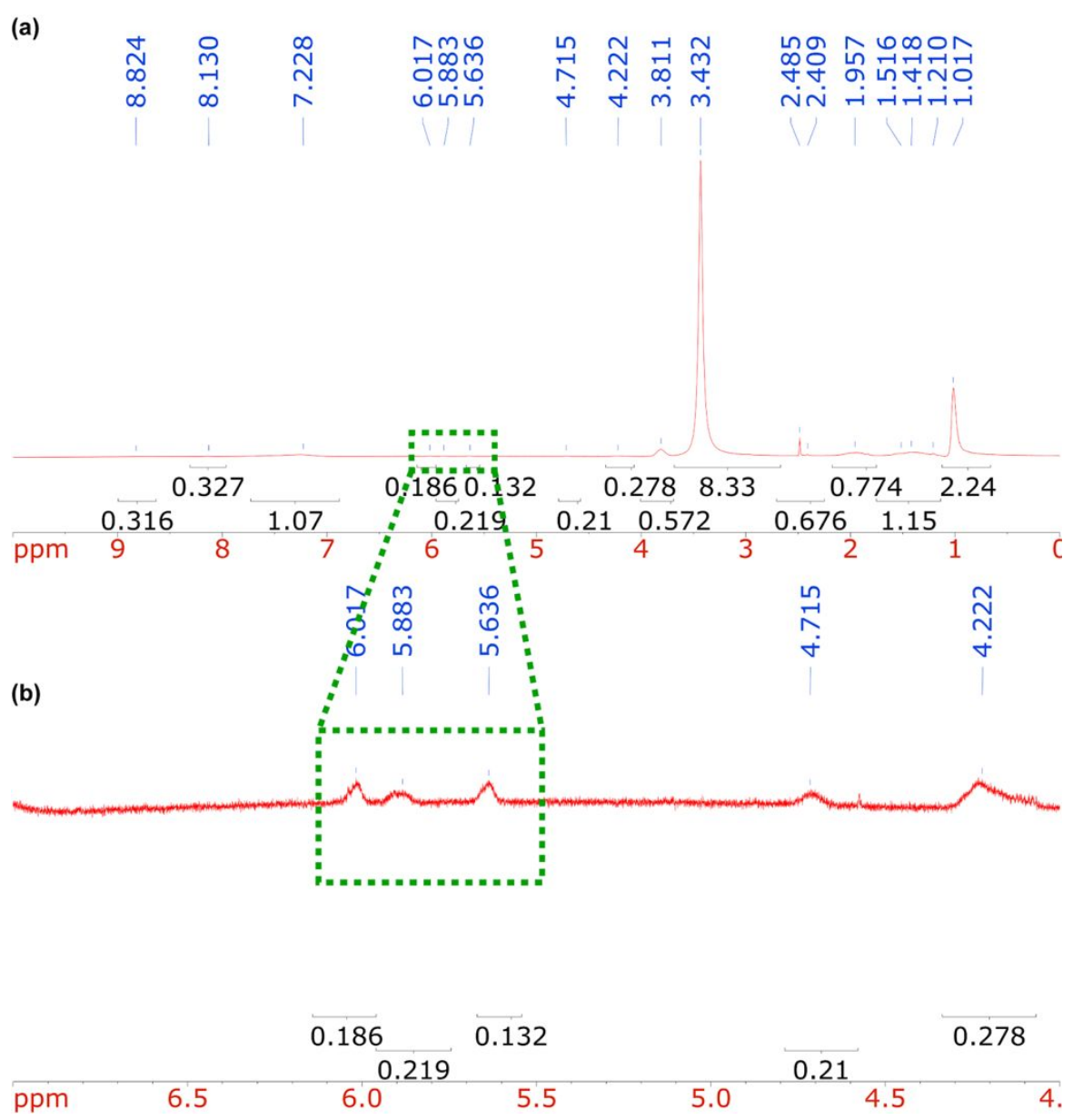

Figure S4 ${ }^{1} \mathrm{H}$ NMR spectrum of HEMA-modified linear pNIPAAm-AAc-Ruthenium polymer. (a) Full spectrum of the HEMA-modified pNIPAAm-AAc-Ruthenium. (b) Enlarged spectrum in the range between 4.0 and $7.0 \mathrm{ppm}$. The peaks between $5.6 \mathrm{ppm}$ and $6.1 \mathrm{ppm}$ indicate that the vinyl groups from HEMA were successfully covalently attached onto the linear polymer skeleton. ${ }^{2}$ 


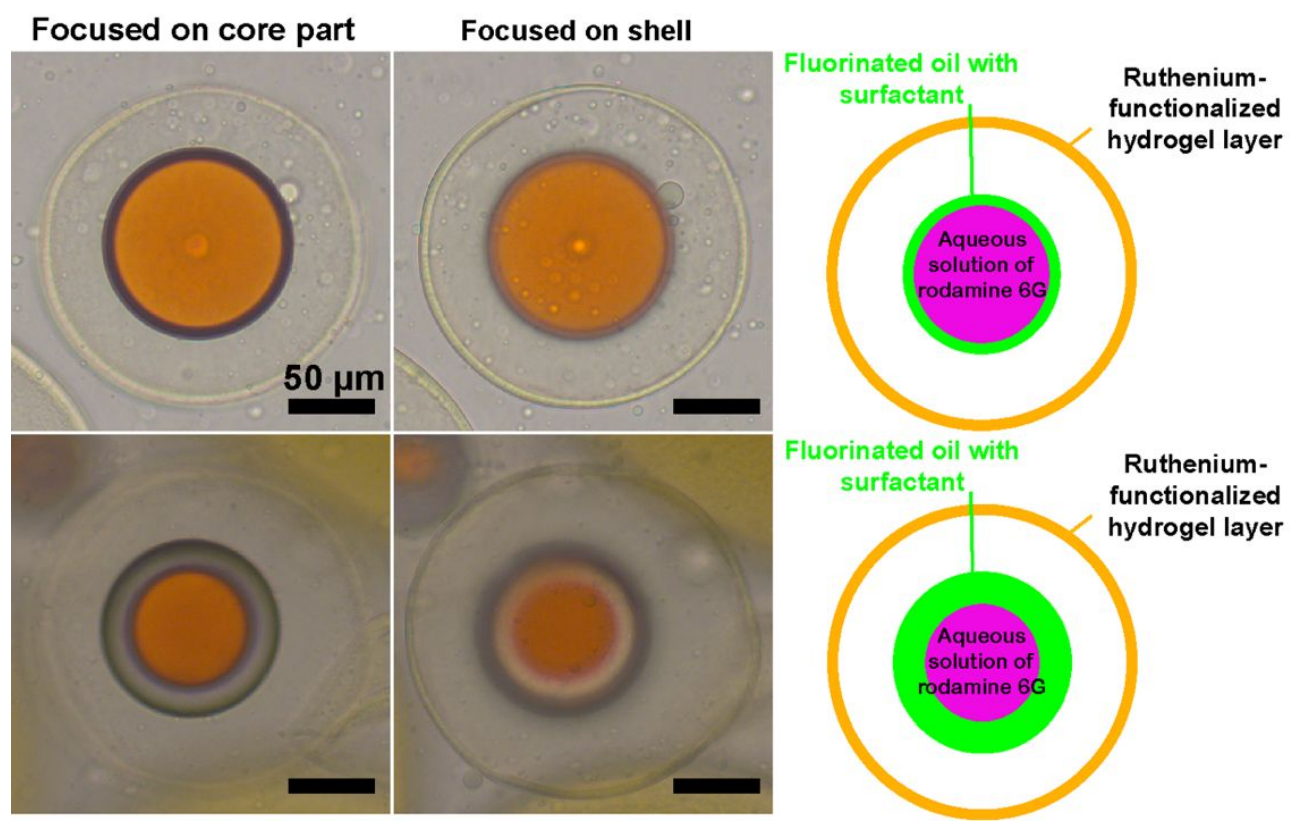

Figure S5 Top: Optical microscopy image of hydrogel microcapsule with thin shells and its cartoon figure (both the external hydrogel shell and the inner fluorinate oil shell). Down: Optical microscopy image of hydrogel microcapsule with thin external hydrogel shell and a relative thick fluorinate oil layer and its cartoon figure (both the external hydrogel shell and the inner fluorinate oil layer). All scale bars are $50 \mu \mathrm{m}$. 

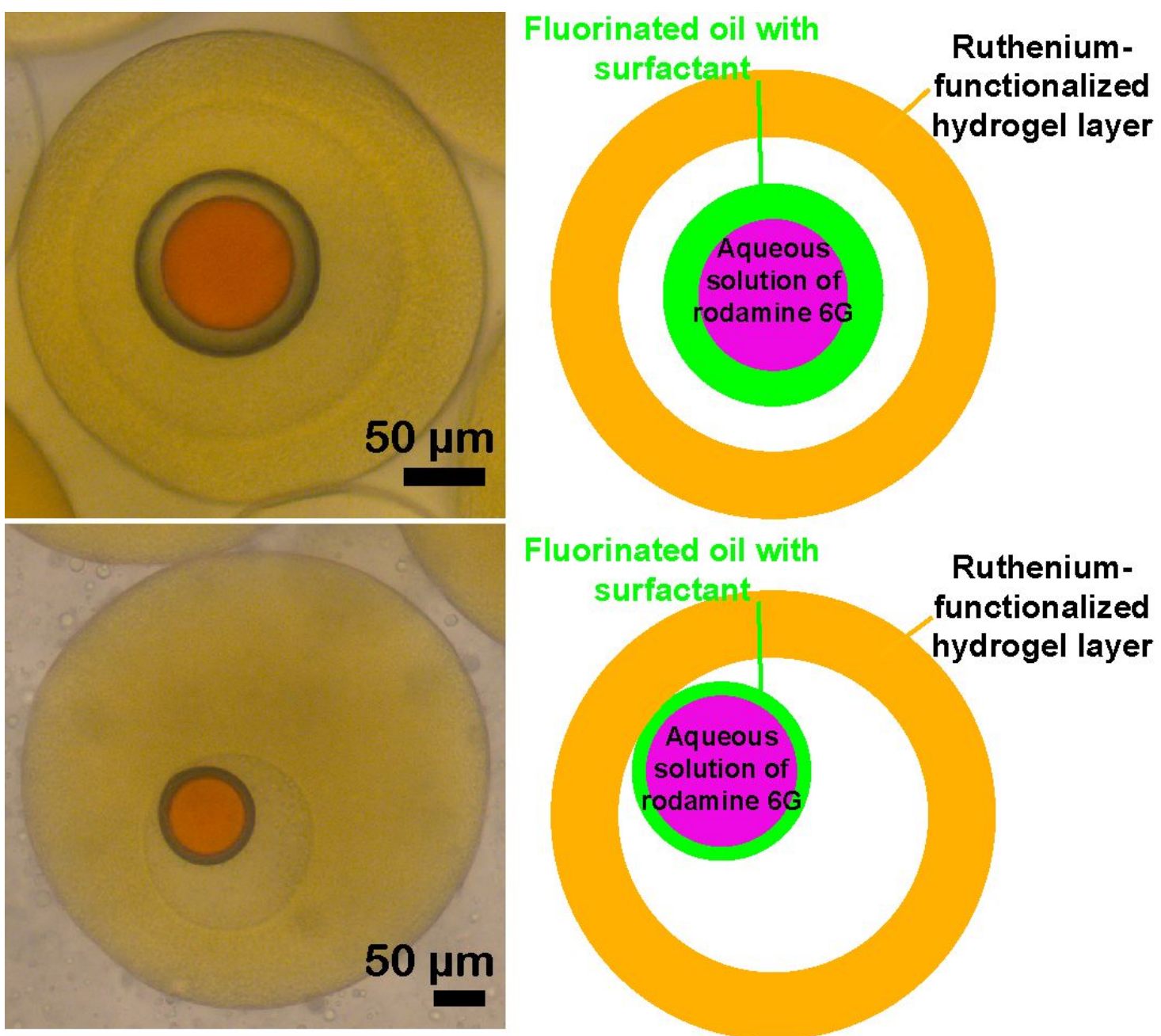

Figure S6 Top: Optical microscopy image of hydrogel microcapsule with thick shells and its cartoon figure (both the external hydrogel shell and the inner fluorinate oil shell). Bottom: Optical microscopy image of hydrogel microcapsule with thick external hydrogel shell and a relative thin fluorinate oil shell and its cartoon figure. 


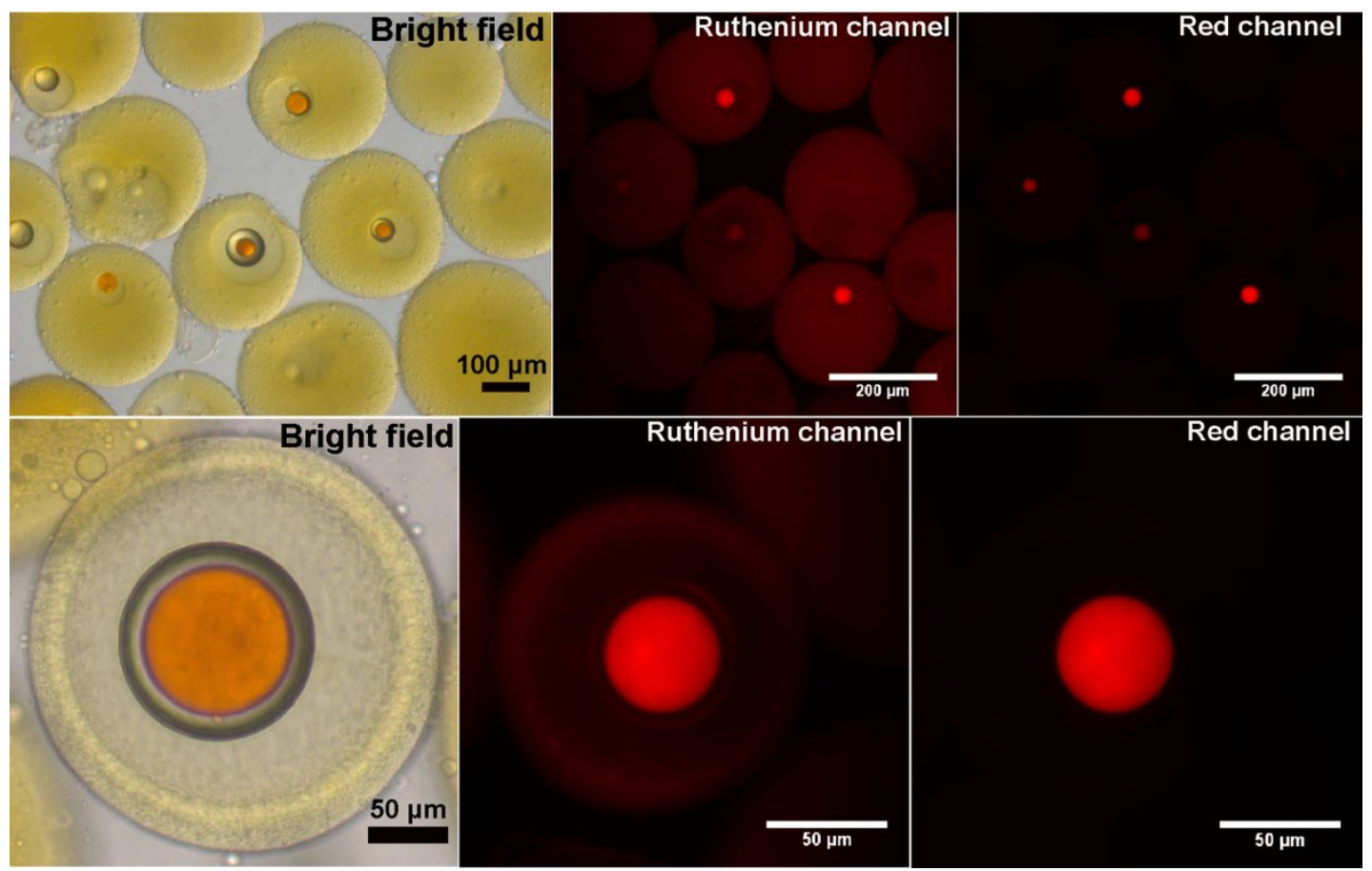

Figure S7 Top: Optical microscopy image of a population of hydrogel microcapsules and the fluorescence microscopy images under different excitation modes. Bottom: Optical microscopy image of a hydrogel microcapsule and the fluorescence microscopy image under different modes. Specifically, the fluorescence microscopy image under red channel shows that all of the rhodamine $6 \mathrm{G}$ was retained inside the inner core part, which was separated by a fluorinated oil layer from outer hydrogel shell and external environment. 


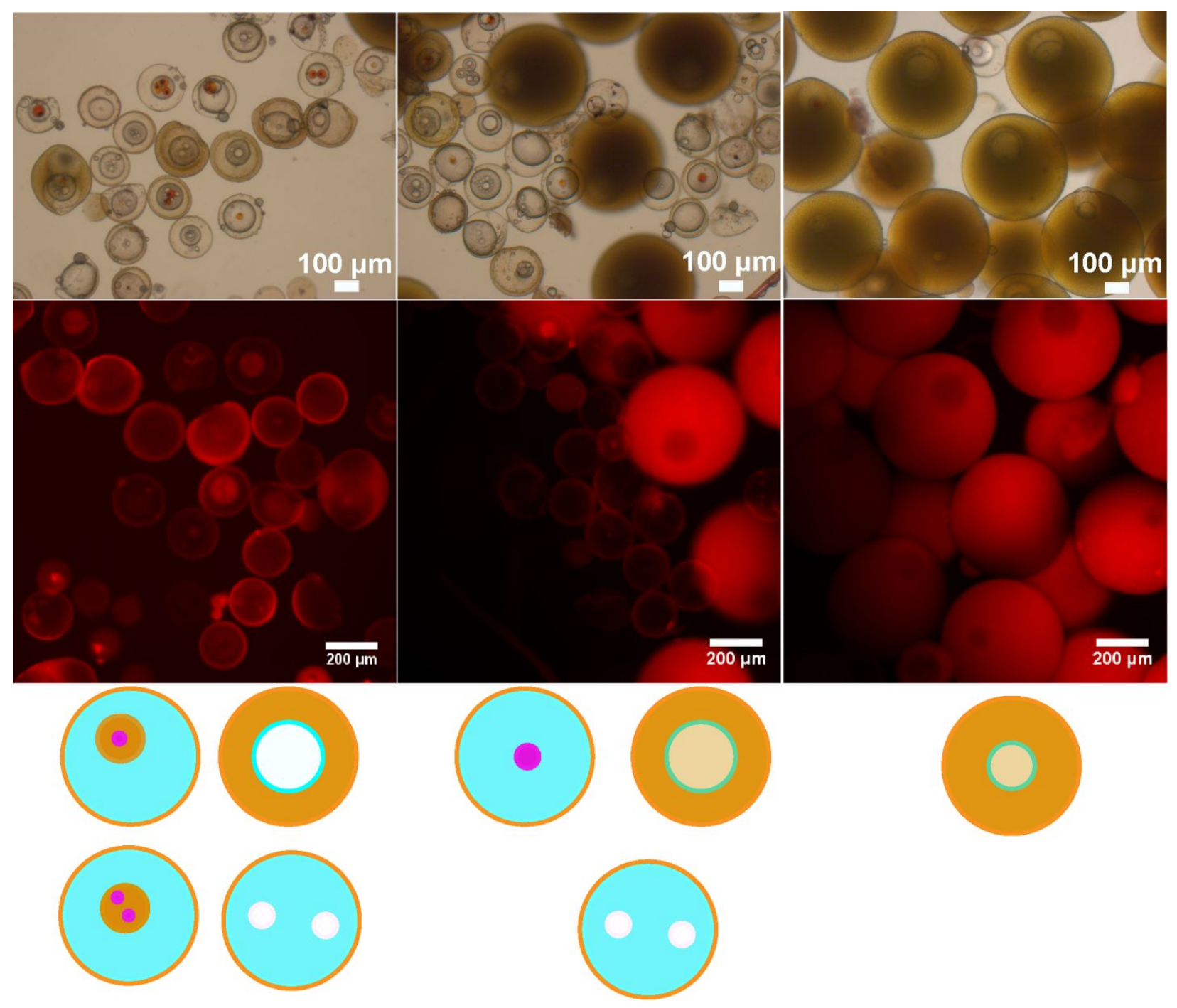

Figure S8 Top row: Optical microscopy image of a population of hydrogel microcapsules.

Middle row: Fluorescence microscopy image of the hydrogel microcapsules corresponding to the top row. The fluorescence microscopy images were all taken under ruthenium mode. Bottom row: Cartoon figure of the hydrogel microcapsules. The microcapsules were in distilled water solution. 

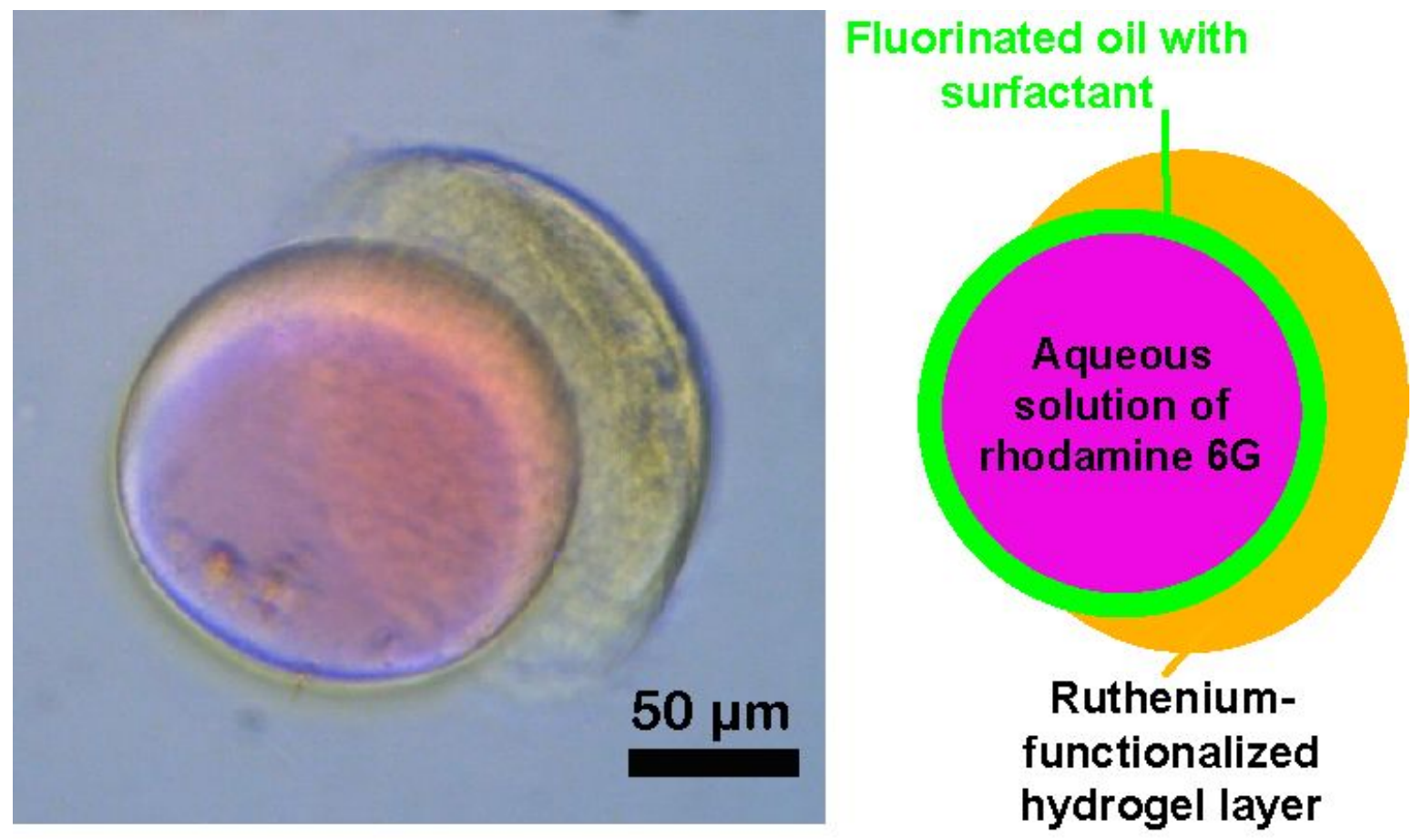

Figure S9 Left. Optical microscopy image shows a Janus micro object in aqueous solution. The left side of the object is an aqueous solution of rhodamine $6 \mathrm{G}$ droplet in perfluorinated oil while the right side is ruthenium-functionalized hydrogel part. The formation of this Janus micro object was as a result of dewetting process took place before the droplet template was completely solidified under UV illumination. 

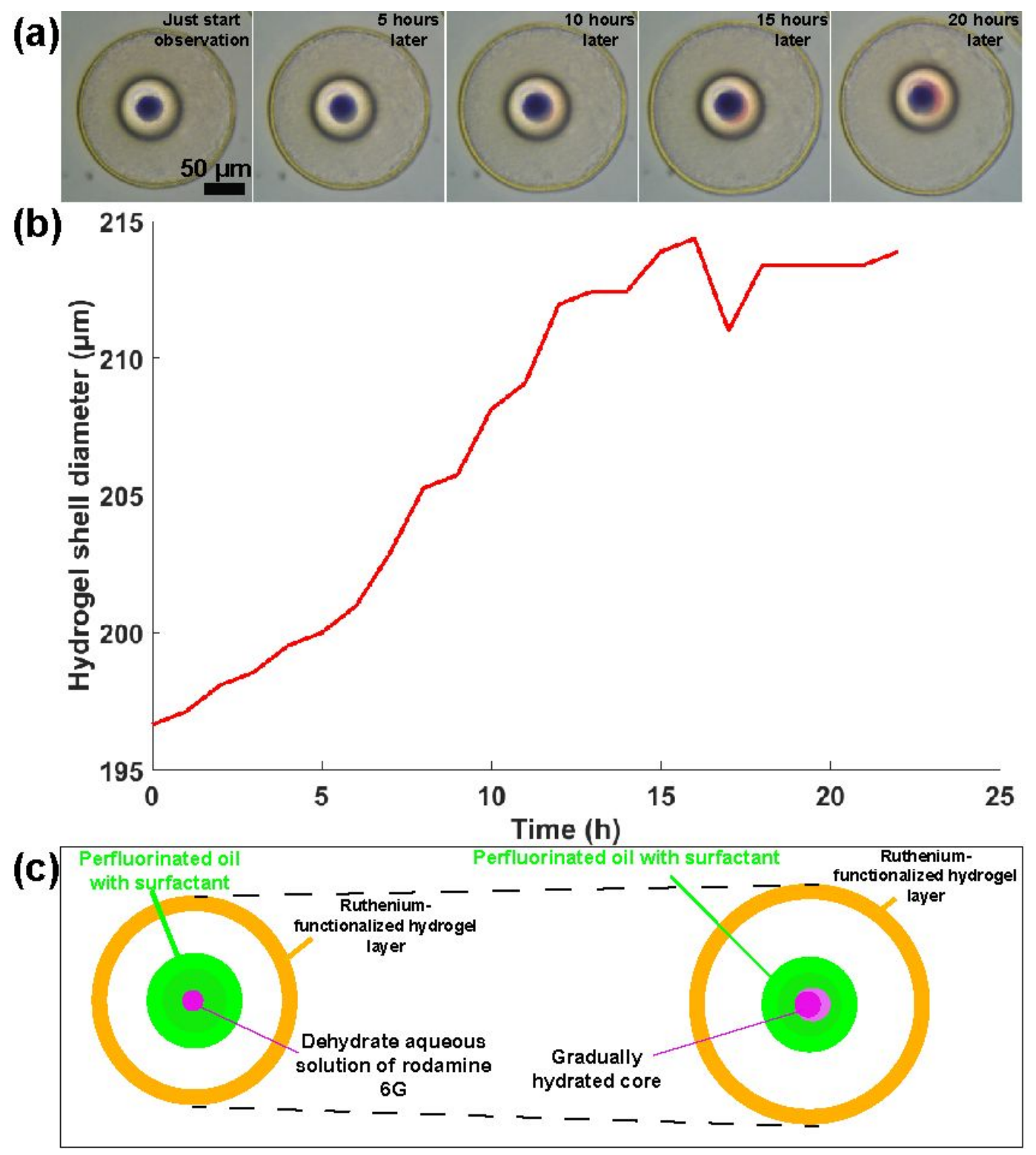

Figure S10 (a) Optical microscopy image of the first 20 hours of evolution of a hydrogel microcapsule with dehydrated core in distilled water. (b) The change of the diameter of hydrogel microcapsule as a function of time. (c) Illustration figure shows how the hydrogel microcapsule evolves in distilled water. The result indicates that microcapsule with a dehydrated core could further prolong the storage period for small molecules when the microcapsule is placed in water solution. The scale bar in (a) is applicable to all five photos. 


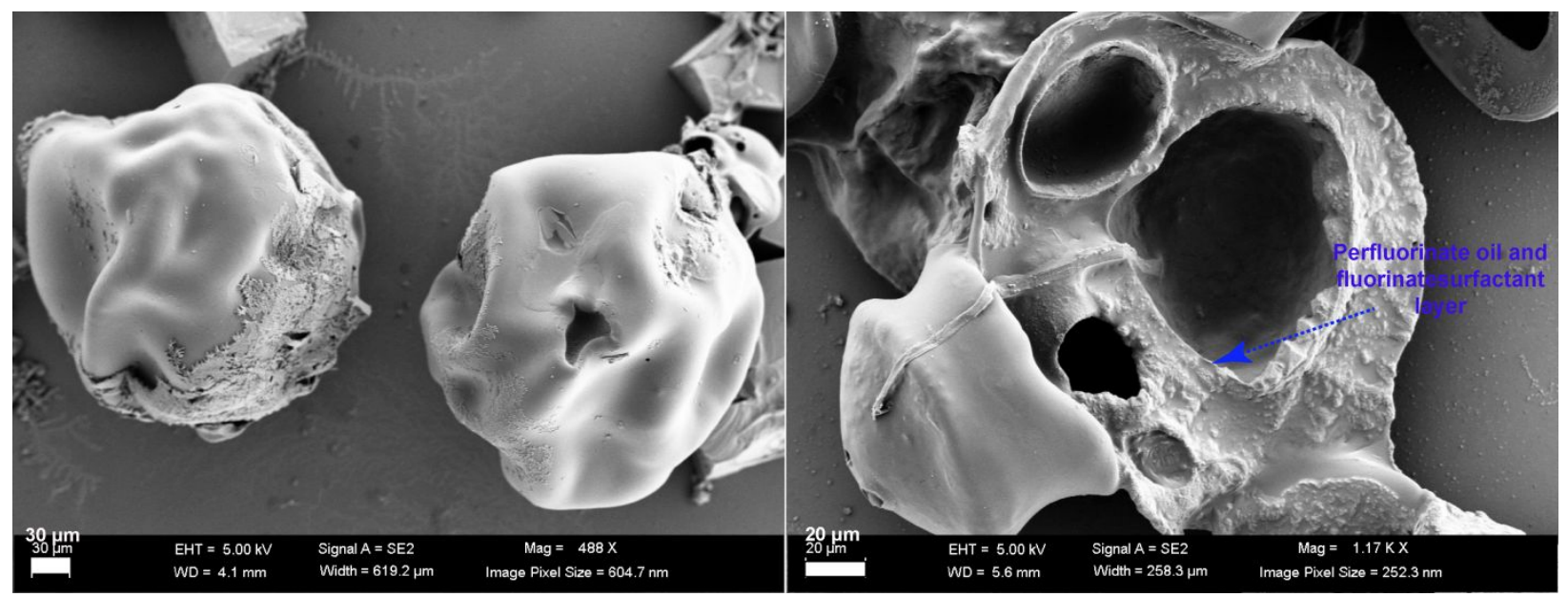

Figure S11 Scanning electronic microscopy of hydrogel microcapsules: Left. Morphology of the microcapsules; Right. Cross-section image of a microcapsule with dual cores.

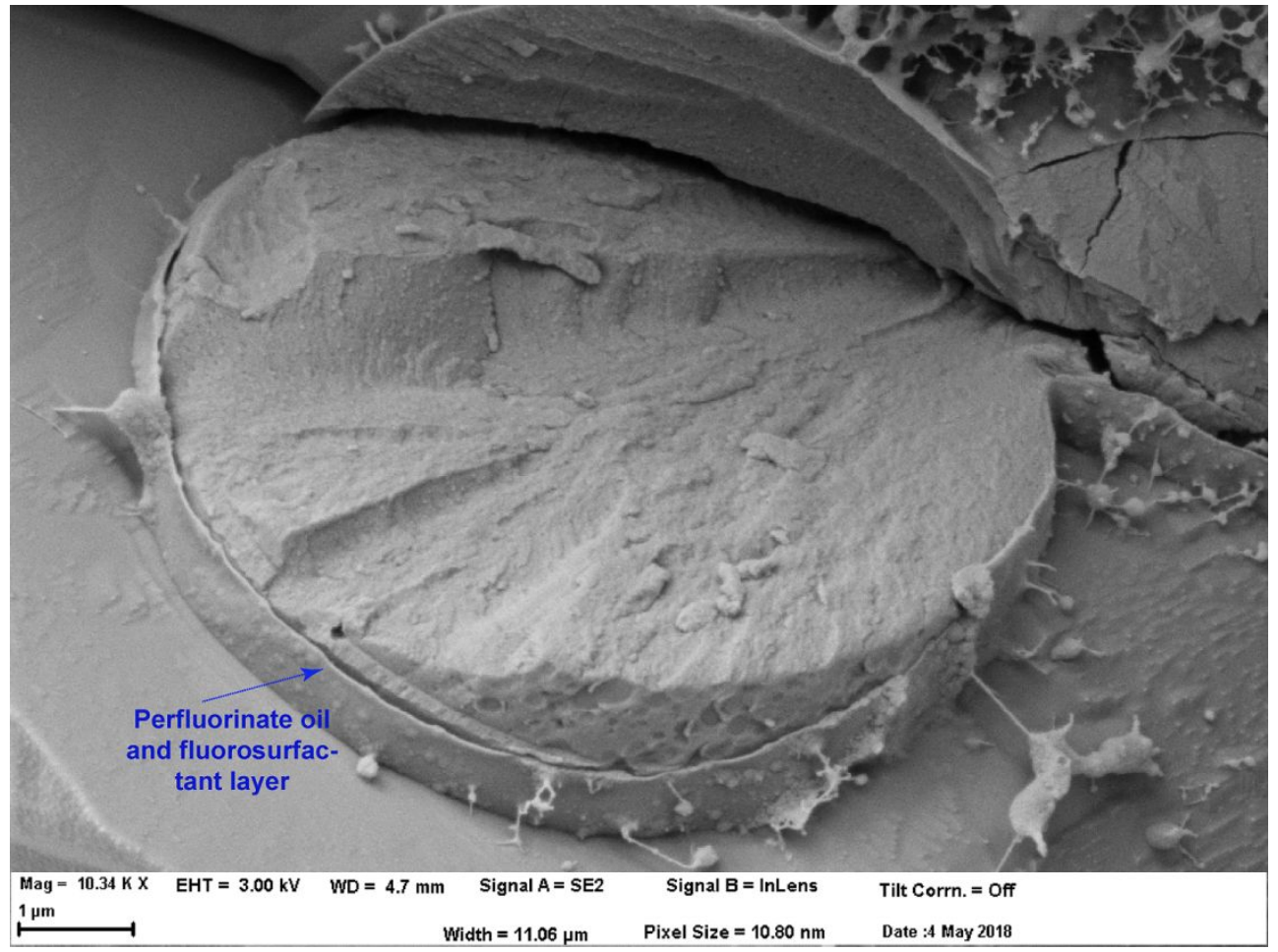

Figure S12 Cryo-SEM of cross-section of a microcapsule with only perfluorinate oil layer, which was presumably formed from the dewetting process that the hydrogel shell could not successfully encapsulate the perfluorinate oil layer. 


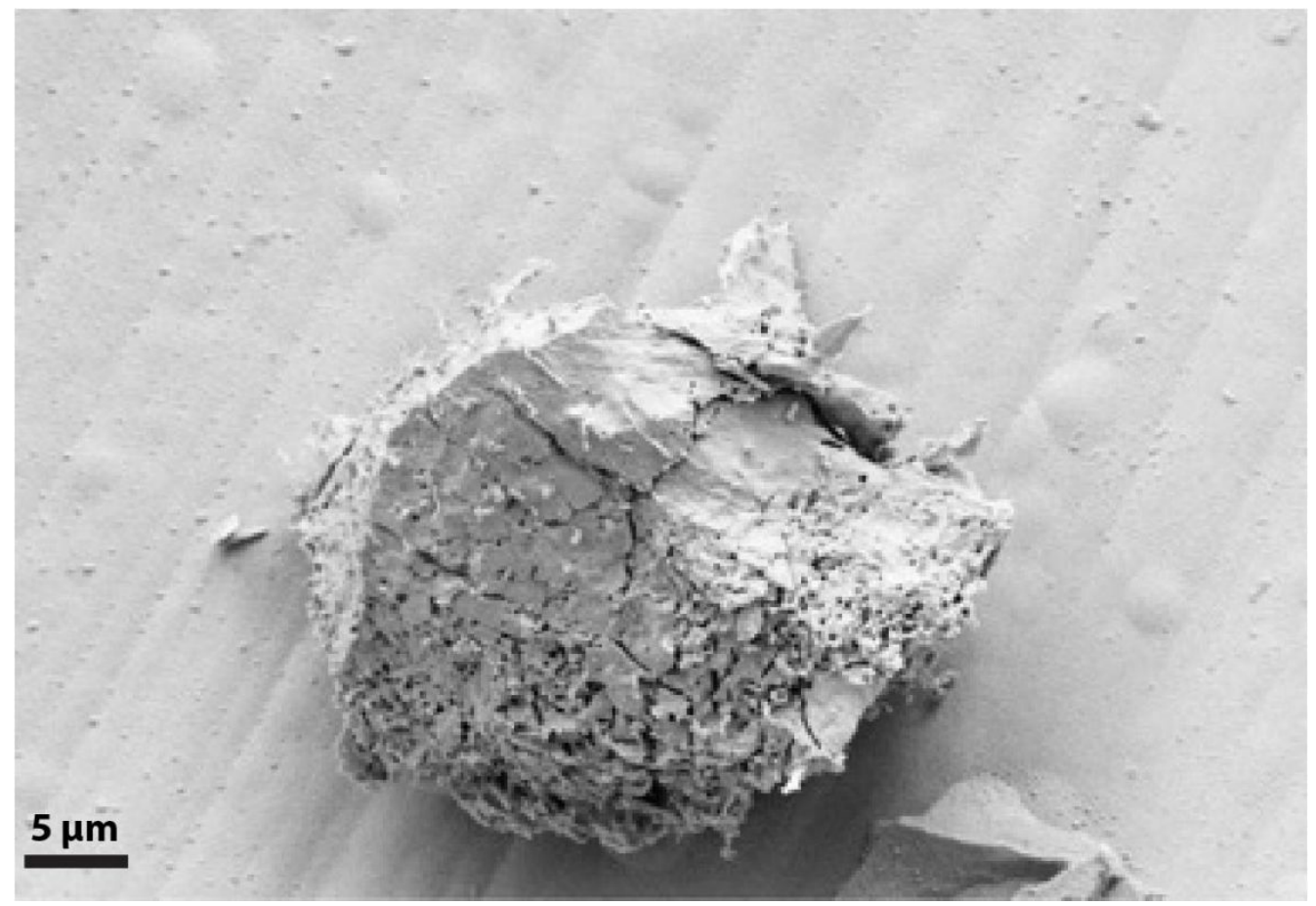

Figure S13 Cryo-SEM of a piece of hydrogel shell from the hydrogel microcapsules. The image reveals that the hydrogel shell material contains many porous structures.
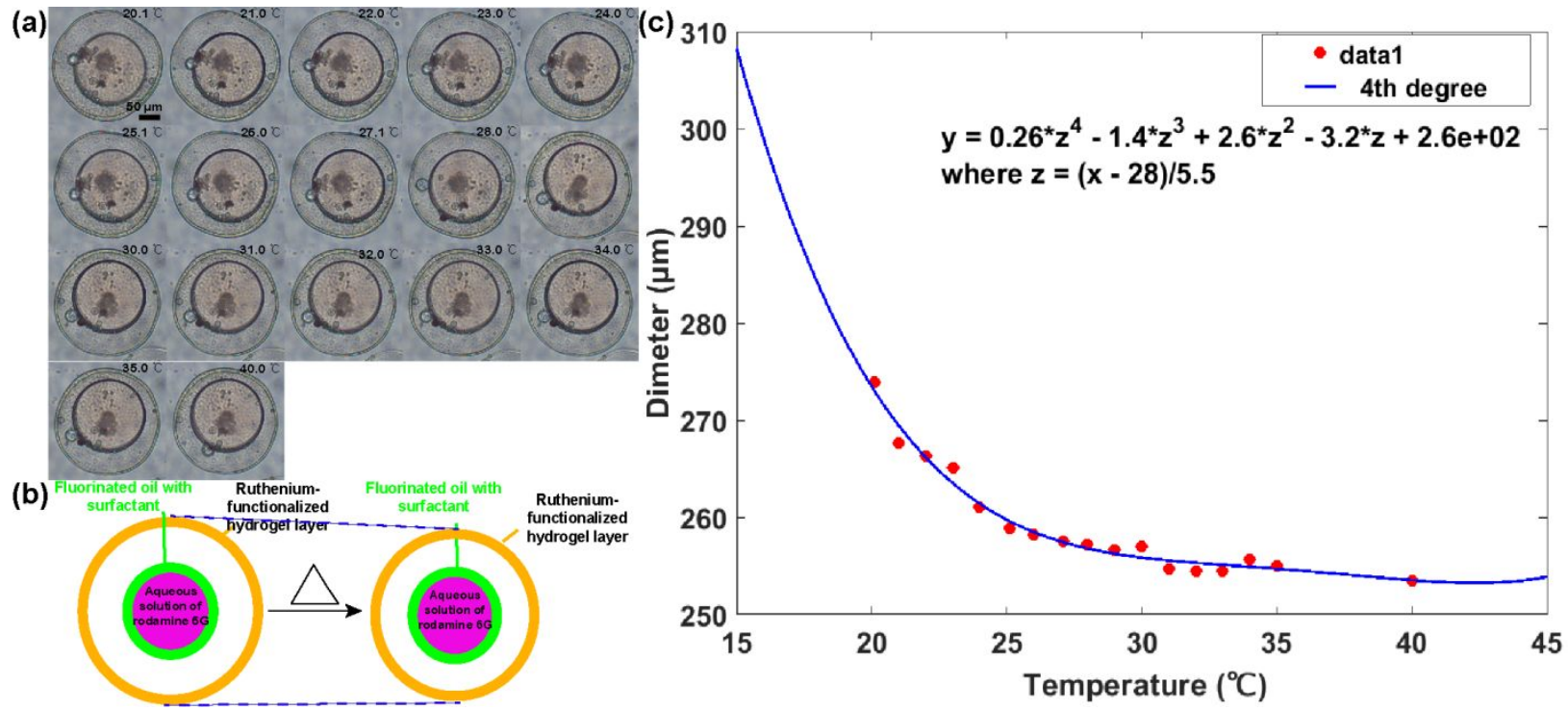

Figure S14 Thermoresponsive property of a hydrogel microcapsule. (a) Optical microscopy images of the hydrogel microcapsule under different temperatures varied from $20.1{ }^{\circ} \mathrm{C}$ to $40.0{ }^{\circ} \mathrm{C}$. 
(b) Illustration figure depicts the evolution of the microcapsule under temperature stimulus. (c)

Plot shows the change of the outer hydrogel shell as a function of temperature.

For calculations of the dilution of core parts of the microcapsules:

\begin{tabular}{|c|c|c|c|c|c|}
\hline $\begin{array}{c}\text { Number of } \\
\text { Figure }\end{array}$ & $\begin{array}{c}\text { Diameter of } \\
\text { core part } d_{i} \\
(\text { Initial }) \\
(\mu \mathrm{m})\end{array}$ & $\begin{array}{c}\text { Diameter of } \\
\text { core part } d_{f} \\
(\text { Final }) \\
(\mu \mathrm{m})\end{array}$ & $\begin{array}{c}\text { Volume of } \\
\text { core part } V_{i} \\
(\text { Initial) } \\
\left(\mu \mathrm{m}^{3}\right)\end{array}$ & $\begin{array}{c}\text { Volume of } \\
\text { core part } V_{f} \\
(\text { Final) } \\
\left(\mu \mathrm{m}^{3}\right)\end{array}$ & $\begin{array}{c}\text { Dilution of } \\
\text { the core part } \\
\text { solution }\left(V_{f} /\right. \\
\left.V_{i}\right)\end{array}$ \\
\hline Figure 3 (a) & 62.7 & 101.7 & $1.29 * 10^{5}$ & $5.51 * 10^{5}$ & 4.27 \\
\hline Figure 4 (a) & 44.1 & 76.1 & $4.49 * 10^{4}$ & $2.31 * 10^{5}$ & 5.14 \\
\hline Figure 5 (c)-(f) & 40.7 & 127.5 & $3.53 * 10^{4}$ & $1.09 * 10^{6}$ & 30.8 \\
\hline \multicolumn{7}{r}{ Where $V_{i}=\frac{4}{3} \pi\left(\frac{d_{i}}{2}\right)^{3}$ and $V_{i}=\frac{4}{3} \pi\left(\frac{d_{f}}{2}\right)^{3}$} \\
\end{tabular}

Table S1. Calculated dilution of the microcapsules under different conditions from Figure 3 to Figure 5.

\section{References:}

(1). Safety Assessment of Polyoxyalkylene Siloxane Copolymers, Alkyl-Polyoxyalkylene Siloxane Copolymers, and Related Ingredients as Used in Cosmetics. Cosmetic Ingredient Review, 2014. May 16, 2014. https://www.cirsafety.org/sites/default/files/alkoxy\%20polysiloxanes.pdf

(2). Chen, M.; Zhou, L.; Guan, Y.; Zhang, Y., Polymerized Microgel Colloidal Crystals: Photonic Hydrogels with Tunable Band Gaps and Fast Response Rates. Angew. Chem. 2013, 52 (38), 9961-5. 